\title{
Cultivar Differentiation Identified by SSR markers and the Application for Polyploid Loquat Plants
}

\author{
Manabu Watanabe ${ }^{1 *}$, Toshiya Yamamoto ${ }^{2}$, Mari Ohara $^{1}$, Chikako Nishitani \\ and Shigeki Yahata ${ }^{3}$ \\ ${ }^{1}$ Chiba Prefectural Agriculture and Forestry Research Center, 808 Daizenno, Midori-ku, Chiba 266-0006, Japan \\ ${ }^{2}$ National Institute of Fruit Tree Science, National Agriculture and Food Research Organization, 2-1 Fujimoto, Tsukuba 305-8605, \\ Japan \\ ${ }^{3}$ Southern Prefectural Horticulture Institute, Chiba Prefectural Agriculture and Forestry Research Center, 1762 Yamamoto, Tateyama \\ 294-0014, Japan
}

Here we established a DNA marker-based method to identify cultivars of loquat (Eriobotrya japonica Lindl.) using simple sequence repeat (SSR) markers derived from pears and apples. A total of 24 loquat cultivars commercially grown in Japan were used for genetic identification, including 15 diploid-, 6 triploid-, and 3 tetraploid cultivars. Analysis using the 88 SSR markers derived from pears and apples, which belong to the same subfamily (family Rosaceae, subfamily Maloideae) as the loquat, indicated that 26 SSR markers were applicable to the identification of loquat cultivars. A total of 82 putative SSR alleles were obtained by the 26 SSR markers. SSR analysis enabled the identification of all loquat varieties tested, except for 'Tomihusa' and '4N-Tomihusa'. In addition, the parentages of several cultivars, including 'Oohusa' and 'Mizuho', were confirmed from the SSR genotype data obtained. Triploid and tetraploid varieties could be identified from the SSR genotypes because some SSR loci generated 2 or more alleles for polyploids. The relationship between 'Kibou' and its parents was confirmed since SSR alleles of the seedless triploid offspring 'Kibou' were clearly inherited from the female parent ' $4 \mathrm{~N}$-Tanaka 1' and the male parent 'Nagasakiwase'. The phenogram obtained by cluster analysis showed no distinctive separation of Japanese commercial cultivars from Chinese cultivars. This result was consistent with the hypothesis that Japanese commercial cultivars were derived from introduced Chinese loquat.

Key Words: DNA marker, Eriobotrya japonica, simple sequence repeat, triploid, tetraploid.

\section{Introduction}

The loquat (Eriobotrya japonica Lindl.) is an evergreen fruit tree, belonging to the family Rosaceae, subfamily Maloideae, and is widely cultivated in Asia, including China and Japan, and in Europe, especially in coastal areas of the Mediterranean. The genus Eriobotrya has about 20 species with wide ranges in the temperate and subtropical regions of China and southeastern Asia, centered in the upper Yangtze River area in Szechwan (Sichuan) Province. Among the Eriobotrya species, only one, E.japonica, is edible. In China, where E.japonica originated, many cultivars were developed in the 7 th century and the loquat was established as one of the major fruit tree species (Ichinose, 2002; Yu, 1979). A

Received; January 10, 2008. Accepted; April 15, 2008

* Corresponding author (E-mail: m.wtnb38@ma.pref.chiba.lg.jp). temperate climate, with mild temperatures and no extreme temperature changes, is considered to be suitable for the cultivation of loquats. International data show that loquats are primarily grown in Asia (China 42,000 ha, Japan 2,420 ha, Pakistan 11,000 ha) followed by the Mediterranean coastal regions (Spain 2,914 ha, Turkey 1,470 ha, Italy 663 ha, Israel 330 ha) and South America (Brazil 300 ha) (Caballero et al., 2002).

In Japan, 'Mogi', 'Tanaka', and 'Kusunoki', which are the current major commercial cultivars, were developed from seedlings of Chinese varieties in the early 19th century. This led to full-scale cultivation of the loquat in Japan. Many of the loquat cultivars that are commercially cultivated in Japan were developed by hybridization using 'Mogi' or 'Tanaka'. Early-maturing cultivars 'Mogi' and 'Nagasakiwase' are cultivated in Kyushu (e.g., Nagasaki Prefecture and Kagoshima Prefecture) and the middle- to late-maturing cultivars 
'Oohusa' and 'Tanaka' are cultured in Honshu and Shikoku (e. g., Chiba Prefecture, Kagawa Prefecture, and Ehime Prefecture).

In recent years, superior loquat cultivars with big fruit, high fruit quality, and high production have been developed; however, as the loquat can be clonally propagated by grafting, the unlawful use or violation of rights concerning these new cultivars is feared. As the confirmation of illegal activities by cultivation testing is timeconsuming in fruit trees, including the loquat, the identification of cultivars is generally carried out by analyzing phenotypes. However, phenotypes can be affected by environmental factors and the exact identification of cultivars is difficult even for experienced experts involved in the production and distribution of loquats; therefore, a scientific method for identifying cultivars has been strongly hoped for. In addition, in the case of fresh fruit, a method of rapid identification is necessary to prevent the loss of profits that occurs during standard identification procedures. Techniques using DNA markers are promising, as they can be rapid, reliable, and objective.

The identification of loquat cultivars using DNA markers such as random amplified polymorphic DNA (RAPD) has been reported. Vilanova et al. (2001) analyzed 33 cultivars, including those cultivated in Spain by the RAPD method, and observed 29 polymorphic bands. They reported that 22 cultivars could be identified among 33 cultivars. Fukuda et al. (2002) showed that 69 varieties, including cultivars introduced from Mexico and China, could be identified by 108 polymorphic bands observed in RAPD analysis. Badenes et al. (2003) showed rather narrow genetic diversity in the loquat varieties cultivated in Europe by using RAPD and simple sequence repeat (SSR) markers. They also reported the usefulness of SSR markers. Soriano et al. (2005), in a study to identify 40 cultivars using SSR markers, reported that the cultivars tested could be classified into 34 genotypes by 39 alleles. In spite of these findings, the identification of cultivars by DNA markers remains in the preliminary stage, and further developments using reliable DNA markers are necessary.

Many major fruit tree species, such as the Japanese pear (Pyrus pyrifolia Nakai) and the apple (Malus domestica Borkh.), as well as the loquat, belong to family Rosaceae, subfamily Maloideae. The applicability of SSR markers developed for the apple and the Japanese pear has been reported (Liebhard et al., 2002; Yamamoto et al., 2001, 2002c, 2004). Yamamoto et al. (2001, 2002c) also indicated that apple-derived SSR markers could be used for linkage map construction and genetic analysis. Liebhard et al. (2002) further reported that apple-derived SSR markers could be applied widely to plants belonging to subfamily Maloideae, such as the loquat. In addition, SSR markers derived from the apple and the Japanese pear could be used effectively to identify quince cultivars and determine parentage relationships (Yamamoto et al., 2004).
To develop a reliable DNA technique for the identification of loquat cultivars, the applicability of SSR markers developed in apples and the Japanese pear was tested. Identification of polymorphisms with respect to SSR markers, confirmation of the parent-offspring relationship, analyses of triploid and tetraploid strains, and genotyping of the triploid seedless cultivar 'Kibou' are reported.

\section{Materials and Methods}

\section{Plant materials}

The plants used in this study were: 15 major diploid cultivars commercially grown in Japan, which had been maintained in the Southern Prefectural Horticulture Institute, Chiba Prefectural Agriculture and Forestry Research Center; 6 triploid varieties, including 'Kibou' and its siblings; and 3 tetraploid varieties, including a parent of 'Kibou' (Table 1). '4N-Tanaka 1' and ' $3 \mathrm{~N}$ Tanaka seedling' were kindly supplied by Mr. Muranishi (South Kyushu University) as scions. The other polyploids were bred and obtained from the Southern Prefectural Horticulture Institute, Chiba Prefectural Agriculture and Forestry Research Center. The tetraploid strains either spontaneously formed or were induced by colchicines treatment of the apical buds of trees or seedlings (Yahata et al., 2004). The triploid varieties were bred by hybridizing tetraploid seed parents with diploid cultivars. The ploidy of these varieties was confirmed by flow cytometry (Ploidy Analyzer FAS Type, Ikeda Scientific Co., Tokyo, Japan).

\section{Extraction of DNA}

DNA was extracted from the leaves immediately after their unfolding. Samples for DNA extraction were $0.05 \mathrm{~g}$ of leaves taken from each variety. Genomic DNA was extracted using the Nucleon Phytopure DNA extraction kit (GE Healthcare UK Ltd., Buckinghamshire, England). To avoid high viscosity and coloring, which were probably due to contaminants, Reagent 1 in the kit was modified by adding mercaptoethanol $(1 \%(\mathrm{v} / \mathrm{v}))$, pectinase $(0.5 \%(\mathrm{w} / \mathrm{v}))$, and $\alpha$-amylase $(135 \mathrm{U} / \mathrm{mL})$ just before application. Concentrations of DNA were determined, after $0.8 \%(\mathrm{w} / \mathrm{v})$ agarose gel electrophoresis and ethidium bromide staining, by comparison with $\lambda$ DNA of known concentrations under UV irradiation.

\section{SSR analysis}

A total of 88 SSR markers were used: 43 markers derived from Japanese pears (Sawamura et al., 2004; Yamamoto et al., 2002a, b, c; Table 3) and 45 markers derived from apples (Liebhard et al., 2002) (Table 2). PCR was carried out in a reaction volume of $20 \mu \mathrm{L}$, with $5 \mathrm{ng}$ of DNA extracted from the loquat varieties being used as a template, 5'-fluorescence-labeled (Fam/Vic/ Ned) primer pair $(250 \mathrm{nM}, 0.2 \mathrm{mM}$ dNTP (Takara Co., Ohtsu, Japan), $1.5 \mathrm{mM} \mathrm{MgCl} 2$ (Takara Co.), and $1 \mathrm{U}$ of Taq polymerase (Takara Co.). The reaction mixture was 
Table 1. Plant materials used in this study.

\begin{tabular}{|c|c|c|}
\hline Variety name & Origin & Ploidy \\
\hline Tanaka & Chance seedlings of Chinese loquat & diploid \\
\hline Oohusa & Tanaka $\times$ Kusunoki & diploid \\
\hline Mizuho & Tanaka $\times$ Kusunoki & diploid \\
\hline Kusunoki & Chance seedlings of Chinese loquat & diploid \\
\hline Nagasakiwase & Mogi $\times$ Hondawase & diploid \\
\hline Toi & Chance seedlings of Chinese loquat & diploid \\
\hline Satomi & Seedlings of Kusunoki & diploid \\
\hline Husahikari & Mizuho $\times$ Tanaka & diploid \\
\hline Tomihusa & Tsukumo $\times$ Mizuho & diploid \\
\hline Husahime & Kusunoki $\times$ Tsukumo & diploid \\
\hline Mogi & Chance seedlings of Chinese loquat & diploid \\
\hline Shiromogi & Gamma-ray irradiation of seedlings of Mogi & diploid \\
\hline Hakugyoku & Introduced from China & diploid \\
\hline Kahou 2 gou & Introduced from China & diploid \\
\hline Daishouhou & Introduced from China & diploid \\
\hline Kibou & 4 N-Tanaka $1 \times$ Nagasakiwase & triploid \\
\hline 3N-Nagasaki 17 & $4 \mathrm{~N}$-Tanaka $1 \times$ Nagasakiwase & triploid \\
\hline 3N-Nagasaki 30 & $4 \mathrm{~N}$-Tanaka $1 \times$ Nagasakiwase & triploid \\
\hline $3 \mathrm{~N}$-Tanaka seedling & $4 \mathrm{~N}$-Tanaka $\times$ Tanaka & triploid \\
\hline 3N-Kusunoki 4 & 4 N-Tanaka $1 \times$ Kusunoki & triploid \\
\hline 3N-Dohi 22 & 4N-Tanaka $1 \times$ Toi & triploid \\
\hline 4N-Tanaka 1 & Colchicine treatment of Tanaka seedlings & tetraploid \\
\hline $4 \mathrm{~N}-\mathrm{So} 75$ & Chance seedlings of Husahikari $\times$ Daishouhou & tetraploid \\
\hline 4N-Tomihusa & Colchicine treatment of shoot buds of Tomihusa & tetraploid \\
\hline
\end{tabular}

put through 35 cycles of $1 \mathrm{~min}$ at $94^{\circ} \mathrm{C}, 1 \mathrm{~min}$ at $52-$ $55^{\circ} \mathrm{C}$ and $2 \mathrm{~min}$ at $72^{\circ} \mathrm{C}$ in the GeneAmp PCR System 9700 (Applied Biosystems, Foster City, CA, USA). The amplification products obtained were separated and detected by a DNA sequencer, ABI PRISM 377 (Applied Biosystems). The fragment lengths of the amplification products were analyzed by the fragment analysis program GeneScan (Applied Biosystems) using fluorescence-labeled DNA molecular-weight markers (400HD-ROX, Applied Biosystems) as internal standards.

\section{Analysis of genotype data}

The presence (1) and absence ( 0 ) of the amplification products were scored for all alleles obtained from the 28 SSR loci in each cultivar or strain. A similarity matrix was prepared from the scored data. Nei's degrees of genetic identity (Nei, 1972) between two cultivars were calculated. A phenogram was constructed from cluster analysis by UPGMA (unweighted pair-group method using arithmetic averages) using the NTSYS-pc program (Rohlf, 2005).

\section{Results and Discussion}

SSR amplification in loquat

In the experimental application of 43 SSR markers derived from Japanese pears, 20 markers produced stable amplified bands. Among them, 12 markers showed polymorphism among varieties. Of 45 SSR markers derived from apples, amplified fragments were observed in 25 markers, among which 14 markers showed polymorphism (Table 2). Of the 88 SSR markers derived from pears and apples, 45 SSRs, about half, were applicable to the loquat, of which 26 , about $30 \%$ of the total, showed polymorphism. The ratios of SSR markers applicable to the loquat were not much different between pear-derived and apple-derived markers.

When compared to the observations that all $12 \mathrm{SSR}$ markers derived from apples were applicable to pears (Yamamoto et al., 2001) and that 77 of 118 SSR markers (65\% of the total) derived from apples and pears could be used for quince analysis (Yamamoto et al., 2004), the percentage of SSRs applicable to loquat was somewhat lower. Soriano et al. (2005) reported that 13 of 30 SSR markers originated from apple could be applicable to the loquat. In this study, 14 additional SSR markers derived from apple and 12 SSRs from pear were found to be applicable to the loquat.

Genetic identification of diploid loquat by SSR markers

In genotype analysis of diploid loquat cultivars using 26 SSR markers, 1 or 2 stable amplification bands were obtained in diploid materials. This suggested that each of the SSR markers used amplified putative alleles derived from a single locus in the genome, as in the case of SSR analysis in the Japanese pear (Kimura et al., 2004). Two to 6 alleles were detected for each SSR marker, totaling 82 alleles. An average of 3.2 alleles was 
detected for each SSR marker. Diploid loquat cultivars could be identified by differences in the responses to plural SSR markers (Table 3).

The parent-offspring relationship was examined in cultivars where the parentages were known. The parentoffspring relationship of 'Oohusa' (offspring) and 'Tanaka' and 'Kusunoki' (parents) was confirmed, with no discrepancy in the alleles found. The parent-offspring relationship between 'Mizuho' and its parents 'Tanaka' and 'Kusunoki' was also confirmed by SSR markers. On the other hand, the parent-offspring relationship between 'Husahikari' (putatively 'Mizuno' $\times$ 'Tanaka'), 'Mizuno' and 'Tanaka' was not supported, as the genetic inheritance of alleles was contradictory. In our attempt to determine the parentage of 'Husahikari', the allele results of 'Kusunoki' $\times$ 'Tanaka' showed no discrepancies; therefore, the possibility that the parents of 'Husahikari' are 'Kusunoki' and 'Tanaka' is high. For each of 'Nagasakiwase', 'Tomihusa', 'Husahime', and 'Kusunoki', one of their parents was included in the varieties tested. As the genetic inheritance of alleles had no inconsistencies, the parent-offspring relationships were confirmed. It was shown that SSR markers could be widely applied across the genus and that they were useful in diagnosing loquat parentage, as has been reported for the Japanese pear (Kimura et al., 2003). Soriano et al. (2005) presented the grouping and relationships among loquat cultivars by SSR markers; however, the parent-offspring relationships were not analyzed. In this study, we showed that SSR markers could be successfully utilized for confirmation of the parent-offspring relationship and origin.

\section{Analysis of triploid and tetraploid loquats}

As in the case of diploid cultivars, by analyzing triploid and tetraploid varieties using 26 SSR markers, SSR genotypes were characterized and all varieties could be identified (Table 3). In the analysis, the parentage relationship between 'Kibou' and its parents ' $4 \mathrm{~N}$-Tanaka 1 ' (우) and 'Nagasakiwase' ( $\sigma^{7}$ ) was confirmed (Yahata et al., 2005). '4N-Tanaka 1' is a colchicine-induced tetraploid variety that showed 1 or 2 alleles in all SSR analyses. 'Kibou' is a triploid cultivar that showed 3 alleles in the analysis with SSR markers NH007b and NH014a. Thus, the triploidy of 'Kibou' was also confirmed by molecular markers. The analysis of genotypes showed that SSR alleles of '4N-Tanaka 1' and 'Nagasakiwase' were transferred to 'Kibou' (Table4). From these data, 'Nagasakiwase' was confirmed to be the pollen parent of 'Kibou', as has been assumed. That parentage of ' $4 \mathrm{~N}-\mathrm{So} 75$ ' is described as 'Husahikari' (우) and 'Daishouhou' $\left(\sigma^{7}\right)$. In this study, a parent-offspring relationship between ' $4 \mathrm{~N}-\mathrm{So} 75$ ' and 'Daishouhou' was rejected because of the discrepancy at the 2 SSRs $\mathrm{NH} 014 \mathrm{a}$ and $\mathrm{CH} 05 \mathrm{~g} 08$, whereas 'Husahikari' shared the same alleles as ' $4 \mathrm{~N}-\mathrm{So} 75$ ' in all tested SSRs.

Table 2. SSR markers used for cross-genus amplification in the loquat.

\begin{tabular}{|c|c|c|c|c|c|}
\hline \multicolumn{4}{|c|}{ SSR name ${ }^{\mathrm{z}}$} & Origin & n References ${ }^{y}$ \\
\hline \multicolumn{5}{|c|}{ 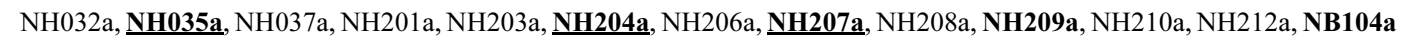 } & $\mathrm{d}$ \\
\hline \multicolumn{5}{|c|}{ NB102a, NB103a, NB105a, NB106a, NB109a, NB110a, NB111a, NB113a, NH019b, NH020a, NH021a } & $\mathrm{c}$ \\
\hline \multicolumn{5}{|c|}{ 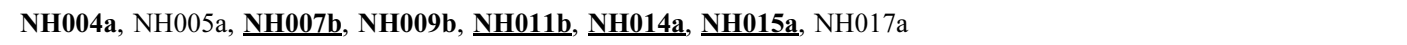 } & $\mathrm{b}$ \\
\hline \multicolumn{5}{|c|}{ BGT23b, BGA35, $\underline{\text { KA14 }}$} & a \\
\hline \multicolumn{5}{|c|}{$\underline{\text { NH033b }}$, NH202a, NH205a, NH211a, NB101a, NB114a, NB135a, $\underline{\text { NB141b }}$} & Table 3 \\
\hline \multicolumn{6}{|c|}{ 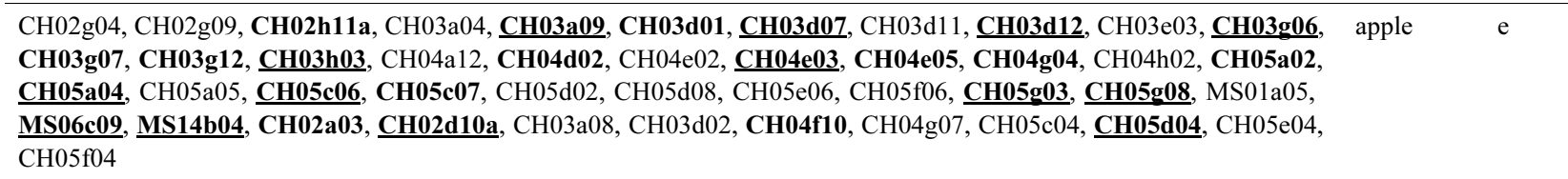 } \\
\hline \multicolumn{6}{|c|}{$\begin{array}{l}\text { z SSR markers showing stable amplification are in bold and polymorphic SSR markers are underlined. } \\
\text { y References for SSR are as follows: a, Yamamoto et al., 2002a; b, Yamamoto et al., 2002b; c, Yamamoto et al., 2002c; d, Sawamura et al., } \\
\text { 2004; e, Liedhard et al., 2002. }\end{array}$} \\
\hline SSR name & Forward primer sequences $\left(5^{\prime}-3^{\prime}\right)$ & Reverse primer sequences $\left(5^{\prime}-3^{\prime}\right)^{z}$ & Origin & Motif & Accession No. \\
\hline NH033b & GTCTGAAACAAAAAGCATCGCAA & CTGCCTCGTCTTCCTCCTTATCTCC & Housui & $(\mathrm{AG})_{22.5}$ & AB302413 \\
\hline $\mathrm{NH} 202 \mathrm{a}$ & ATCGACCAATTCAAAGTCTGAT & gtttcttTAGTCTTCCACAACAAACCCTA & Housui & $(\mathrm{AC})_{7}$ & AB302415 \\
\hline NH205a & ATGTCTCGATATGCGTGCTAGG & gtttcttCTTCAAAGCATTCACGGTAATCA & Housui & $(\mathrm{TA})_{6}(\mathrm{CA})_{8}$ & AB302416 \\
\hline NH211a & GGGAAATTCACACAACTCTTAGGG & gtttcttTTCGAATATGCAAAACAACAAGTG & Housui & $(\mathrm{AC})_{6}$ & AB302417 \\
\hline NB101a & GAAAGAGAAGGATAGCTGGTTA & TTTGCTGCTTGCTTCTGCTT & Bartlett & $(\mathrm{AG})_{27.5}$ & AB302411 \\
\hline NB114a & AAGAAATAAAACCCACAAAGCC & gtttcttTGTCTTCTCTCTCCGCTTATTC & Bartlett & $(\mathrm{GA})_{15}$ & AB302423 \\
\hline NB135a & TGAGAGAAGAACAGCCAATGAT & gtttcttCTCCCACTCAGATCGCTCCT & Bartlett & $(\mathrm{GA})_{22.5}$ & AB302441 \\
\hline NB141b & GGATTGATCGCCTTATGGTTGT & gtttcttCAGAGAAAGACAGAGGTAGAGAGAA & Bartlett & $(\mathrm{AT})_{8}(\mathrm{AG})_{15.5}$ & AB302443 \\
\hline
\end{tabular}

${ }^{\mathrm{z}}$ gtttctt: pig-tail 
Table 4. Genotypes for 24 loquat varieties identified by 26 SSR markers.

\begin{tabular}{|c|c|c|c|c|c|c|c|c|c|c|c|c|c|}
\hline ariety name & CH03a09 & $\mathrm{CH} 03 \mathrm{~d} 07$ & $\mathrm{CH} 03 \mathrm{~d} 12$ & CH03g06 & CH04h03 & $\mathrm{CH} 04 \mathrm{e} 03$ & $\mathrm{CH} 05 \mathrm{a} 04$ & NH033b & NH035a & NH204a & $\mathrm{NH} 207 \mathrm{a}$ & KA14 & NH007b \\
\hline Tanaka & $140 / 150$ & $306 / 308$ & $115 / 140$ & $6 / 178$ & $102 / 102$ & 178/182 & $185 / 185$ & $196 / 198$ & $144 / 144$ & $110 / 110$ & $156 / 156$ & $175 / 175$ & $129 / 147$ \\
\hline Oohusa & $140 / 140$ & $308 / 308$ & $95 / 140$ & $176 / 178$ & $102 / 102$ & $178 / 182$ & $185 / 185$ & $184 / 198$ & $144 / 144$ & $110 / 110$ & $156 / 156$ & $175 / 175$ & $141 / 147$ \\
\hline Mizuho & $140 / 140$ & $306 / 306$ & $95 / 115$ & $176 / 178$ & $102 / 102$ & 178/182 & $185 / 185$ & $184 / 198$ & $144 / 144$ & $110 / 110$ & $156 / 156$ & $175 / 175$ & $129 / 141$ \\
\hline Kusunoki & $140 / 148$ & $306 / 308$ & $95 / 95$ & $178 / 178$ & $102 / 102$ & $182 / 182$ & $185 / 185$ & $184 / 198$ & $144 / 144$ & $110 / 110$ & $156 / 156$ & $173 / 175$ & $141 / 147$ \\
\hline Nagasakiwase & $134 / 140$ & $308 / 314$ & $115 / 138$ & $176 / 180$ & $94 / 94$ & $178 / 182$ & $185 / 187$ & $184 / 196$ & $144 / 144$ & $110 / 114$ & $156 / 156$ & $175 / 175$ & $129 / 141$ \\
\hline Toi & $134 / 152$ & $308 / 314$ & $95 / 115$ & $180 / 180$ & $94 / 94$ & $182 / 182$ & $185 / 185$ & $184 / 196$ & $144 / 144$ & $110 / 110$ & $156 / 158$ & $173 / 173$ & $147 / 147$ \\
\hline Satomi & $140 / 148$ & $306 / 306$ & $95 / 115$ & $178 / 178$ & $102 / 102$ & $182 / 182$ & $185 / 185$ & $184 / 198$ & $144 / 144$ & $110 / 110$ & $156 / 156$ & $173 / 175$ & $147 / 147$ \\
\hline Husahikari & $140 / 140$ & $306 / 306$ & $95 / 115$ & $176 / 178$ & $102 / 102$ & $182 / 182$ & $185 / 185$ & $198 / 198$ & $144 / 144$ & $110 / 110$ & $156 / 156$ & $173 / 175$ & $147 / 147$ \\
\hline Tomihusa & $140 / 140$ & $306 / 308$ & $115 / 115$ & $176 / 180$ & $102 / 102$ & $178 / 182$ & $185 / 185$ & $184 / 196$ & $144 / 144$ & $110 / 110$ & $156 / 156$ & $175 / 175$ & $129 / 129$ \\
\hline Husahime & $140 / 148$ & $306 / 308$ & $95 / 115$ & $178 / 178$ & $102 / 102$ & $178 / 182$ & $185 / 185$ & $184 / 198$ & $144 / 144$ & $110 / 110$ & $156 / 156$ & $175 / 175$ & $141 / 147$ \\
\hline Mogi & $140 / 140$ & $308 / 310$ & $115 / 140$ & $176 / 180$ & $94 / 102$ & $182 / 184$ & $185 / 185$ & $196 / 196$ & $144 / 144$ & $110 / 114$ & $156 / 156$ & $175 / 175$ & $129 / 129$ \\
\hline Shiromogi & $140 / 140$ & $308 / 310$ & $140 / 140$ & $180 / 180$ & $94 / 102$ & $182 / 184$ & $185 / 185$ & $196 / 196$ & $144 / 144$ & $110 / 110$ & $156 / 156$ & $175 / 175$ & $129 / 129$ \\
\hline Hakugyoku & $136 / 140$ & $308 / 316$ & $115 / 140$ & $176 / 180$ & $94 / 102$ & $182 / 182$ & $185 / 185$ & $184 / 184$ & $152 / 152$ & $110 / 114$ & $156 / 158$ & $175 / 175$ & $129 / 141$ \\
\hline Kahou 2 gou & $148 / 150$ & $306 / 308$ & $95 / 121$ & $176 / 180$ & $94 / 102$ & $182 / 182$ & $185 / 185$ & $184 / 196$ & $144 / 144$ & $110 / 114$ & $156 / 158$ & $175 / 175$ & $129 / 129$ \\
\hline Daishouhou & $140 / 148$ & $306 / 306$ & $95 / 140$ & $176 / 178$ & $102 / 102$ & $178 / 182$ & $185 / 185$ & $184 / 198$ & $144 / 144$ & $110 / 110$ & $156 / 156$ & $173 / 175$ & $147 / 147$ \\
\hline Kibou & $140 / 150$ & $306 / 308$ & $115 / 140$ & $178 / 180$ & $94 / 102$ & $178 / 182$ & $185 / 187$ & $184 / 196$ & $144 / 144$ & $110 / 110$ & $156 / 156$ & $175 / 175$ & $129 / 141 / 147$ \\
\hline 3N-Nagasaki 17 & $140 / 150$ & $306 / 308$ & $138 / 140$ & $178 / 180$ & $94 / 102$ & $178 / 182$ & $185 / 185$ & $196 / 196$ & $144 / 144$ & $110 / 114$ & $156 / 156$ & $175 / 175$ & $129 / 147$ \\
\hline 3N-Nagasaki 30 & $134 / 150$ & $306 / 314$ & $115 / 140$ & $178 / 180$ & $94 / 102$ & $178 / 178$ & $185 / 185$ & $184 / 196$ & $144 / 144$ & $110 / 114$ & $156 / 156$ & $175 / 175$ & $129 / 147$ \\
\hline $3 \mathrm{~N}$-Tanaka seedling & $150 / 150$ & $306 / 308$ & $115 / 140$ & $176 / 178$ & $102 / 102$ & $178 / 182$ & $185 / 185$ & $196 / 198$ & $144 / 144$ & $110 / 110$ & $156 / 156$ & $175 / 175$ & $129 / 129$ \\
\hline $3 \mathrm{~N}-$ Kusunoki 4 & $148 / 150$ & $306 / 308$ & $95 / 115 / 140$ & $178 / 178$ & $102 / 102$ & $182 / 182$ & $185 / 185$ & $196 / 198$ & $144 / 144$ & $110 / 110$ & $156 / 156$ & $175 / 175$ & $129 / 141 / 147$ \\
\hline 3N-Dohi 22 & $140 / 150 / 152$ & $306 / 308$ & $95 / 140$ & $178 / 180$ & $94 / 102$ & $178 / 182$ & $185 / 185$ & $196 / 196$ & $144 / 144$ & $110 / 110$ & $156 / 156$ & $173 / 175$ & $129 / 147$ \\
\hline 4N-Tanaka 1 & $140 / 150$ & $306 / 308$ & $115 / 140$ & $178 / 178$ & $102 / 102$ & $178 / 182$ & $185 / 185$ & $196 / 196$ & $144 / 144$ & $110 / 110$ & $156 / 156$ & $175 / 175$ & $129 / 147$ \\
\hline 4N-So 75 & $134 / 140$ & $306 / 314$ & $95 / 140$ & $170 / 176$ & $94 / 102$ & $182 / 182$ & $179 / 185$ & $198 / 198$ & $144 / 152$ & $110 / 114$ & $156 / 156$ & $173 / 175$ & $147 / 147$ \\
\hline 4N-Tomihusa & $140 / 140$ & $306 / 308$ & $115 / 115$ & $176 / 180$ & $102 / 102$ & $178 / 182$ & $185 / 185$ & $184 / 196$ & $144 / 144$ & $110 / 110$ & $156 / 156$ & $175 / 175$ & $129 / 129$ \\
\hline
\end{tabular}

\begin{tabular}{|c|c|c|c|c|c|c|c|c|c|c|c|c|c|}
\hline Variety name & NH011b & NH014a & NB114a & NB141b & $\mathrm{CH} 05 \mathrm{c} 06$ & $\mathrm{CH} 05 \mathrm{~g} 03$ & CH05g08 & MS06c09 & MS14b04 & $\mathrm{CH} 02 \mathrm{~d} 10 \mathrm{a}$ & CH05d04 & NH015a & NB105a \\
\hline naka & $/ 184$ & $164 / 182$ & $133 / 137$ & $3 / 151$ & 10 & $161 / 180$ & 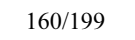 & 41 & $267 / 267$ & $220 / 220$ & $192 / 192$ & $3 / 113$ & tho \\
\hline Dhusa & $/ 184$ & 82 & $3 / 137$ & $/ 151$ & 107/107 & 61 & 99 & 41 & $7 / 267$ & $212 / 220$ & $92 / 192$ & $113 / 119$ & $5 / 165$ \\
\hline Mizuho & $174 / 184$ & $135 / 182$ & $133 / 137$ & $3 / 151$ & $107 / 107$ & $143 / 180$ & & & $7 / 267$ & $212 / 220$ & $2 / 192$ & $113 / 119$ & $5 / 165$ \\
\hline usunoki & $174 / 174$ & $135 / 164$ & $133 / 133$ & $143 / 143$ & 107/109 & $143 / 143$ & $164 / 199$ & $141 / 141$ & $77 / 267$ & $212 / 220$ & $192 / 194$ & $119 / 119$ & $55 / 165$ \\
\hline Nagasakiw: & $174 / 174$ & $135 / 188$ & $133 / 133$ & $143 / 161$ & $107 / 107$ & $143 / 151$ & $164 / 199$ & $119 / 141$ & $67 / 267$ & $212 / 212$ & $192 / 192$ & $113 / 113$ & $55 / 165$ \\
\hline Toi & $174 / 174$ & $135 / 164$ & $137 / 137$ & $143 / 161$ & $109 / 109$ & $161 / 180$ & $160 / 199$ & $149 / 149$ & $273 / 273$ & $216 / 216$ & $192 / 194$ & $113 / 119$ & $155 / 165$ \\
\hline Satomi & $174 / 184$ & $135 / 164$ & $133 / 133$ & $143 / 151$ & $107 / 109$ & $143 / 180$ & $164 / 199$ & $141 / 141$ & $267 / 267$ & $212 / 220$ & $192 / 194$ & $113 / 119$ & $165 / 165$ \\
\hline Husahikari & $174 / 174$ & 135 & 13 & 14 & 107 & 30 & 199 & 41 & $267 / 267$ & $220 / 220$ & $192 / 194$ & $113 / 119$ & $165 / 165$ \\
\hline omihusa & & & & & $107 / 107$ & & & & $267 / 267$ & $216 / 220$ & $192 / 192$ & $113 / 113$ & $165 / 165$ \\
\hline Husahime & $174 / 184$ & $135 / 182$ & & & $107 / 107$ & & & & 267 & $212 / 220$ & $192 / 192$ & $113 / 119$ & $155 / 165$ \\
\hline ogi & & $135 / 188$ & & & $107 / 107$ & & & & 267 & $212 / 216$ & $192 / 192$ & $113 / 113$ & $155 / 165$ \\
\hline iromogi & $186 / 186$ & $135 / 188$ & $133 / 133$ & $143 / 143$ & $107 / 107$ & 14 & $164 / 164$ & 145 & $267 / 267$ & $216 / 216$ & $192 / 192$ & $113 / 113$ & $155 / 165$ \\
\hline Hakugyoku & $174 / 174$ & $135 / 135$ & & & $107 / 107$ & & & & $267 / 273$ & $210 / 220$ & $192 / 194$ & $113 / 113$ & $153 / 165$ \\
\hline Kahou 2 gou & $174 / 174$ & $135 / 182$ & $133 / 137$ & $143 / 143$ & $107 / 109$ & $143 / 143$ & $160 / 160$ & $119 / 119$ & $267 / 267$ & $212 / 216$ & $192 / 194$ & $113 / 113$ & $165 / 165$ \\
\hline Daishouhou & $174 / 174$ & $164 / 164$ & $133 / 137$ & & $107 / 107$ & & & & 267 & $212 / 220$ & 192 & 113 & $155 / 165$ \\
\hline Kibou & $174 / 184$ & $164 / 182 / 188$ & & & $107 / 107$ & & & & 267 & & $192 / 192$ & $113 / 113$ & $165 / 165$ \\
\hline 3N-Nagasaki 17 & $174 / 184$ & $164 / 182 / 188$ & $133 / 137$ & $143 / 151$ & $107 / 107$ & $151 / 161$ & $164 / 199$ & $141 / 141$ & $267 / 267$ & $212 / 220$ & $192 / 192$ & $113 / 113$ & $155 / 165$ \\
\hline 3N-Nagasaki 30 & $174 / 184$ & $164 / 182 / 188$ & $133 / 137$ & $143 / 151$ & $107 / 107$ & $143 / 161$ & $160 / 164 / 199$ & $119 / 141$ & $267 / 267$ & $212 / 220$ & $192 / 192$ & $113 / 113$ & $155 / 165$ \\
\hline 3N-Tanaka seedling & $174 / 184$ & $164 / 182$ & $133 / 137$ & $143 / 151$ & $107 / 107$ & $161 / 180$ & $160 / 199$ & $141 / 141$ & $267 / 267$ & $220 / 220$ & $192 / 192$ & $113 / 113$ & $165 / 165$ \\
\hline 3N-Kusunoki 4 & $174 / 174$ & $135 / 164 / 182$ & $133 / 137$ & $143 / 143$ & $107 / 107$ & $143 / 161 / 180$ & $160 / 199$ & $141 / 141$ & $267 / 267$ & $220 / 220$ & $192 / 194$ & $113 / 119$ & $165 / 165$ \\
\hline 3N-Dohi 22 & $174 / 184$ & $164 / 182$ & $137 / 137$ & $143 / 151$ & $107 / 109$ & $161 / 180$ & $160 / 199$ & $141 / 149$ & $267 / 273$ & $216 / 220$ & $192 / 194$ & $113 / 119$ & $155 / 165$ \\
\hline 4N-Tanaka 1 & $174 / 184$ & $164 / 182$ & $137 / 137$ & $143 / 151$ & $107 / 107$ & $161 / 180$ & $160 / 199$ & $141 / 141$ & $267 / 267$ & $220 / 220$ & $192 / 192$ & $113 / 113$ & $165 / 165$ \\
\hline $4 \mathrm{~N}-\mathrm{So} 75$ & $174 / 174$ & $135 / 135$ & $133 / 137$ & $143 / 161$ & $107 / 122$ & $161 / 180$ & $160 / 179$ & $141 / 145$ & $267 / 273$ & $216 / 220$ & $94 / 194$ & $113 / 113$ & $155 / 165$ \\
\hline 4N-Tomihusa & $174 / 184$ & $182 / 182$ & $133 / 133$ & $151 / 151$ & $107 / 107$ & $151 / 180$ & $164 / 199$ & $141 / 141$ & $267 / 267$ & $216 / 220$ & $192 / 192$ & $113 / 113$ & $165 / 165$ \\
\hline
\end{tabular}

Genetic relatedness analyzed by SSR markers

A phenogram of the 15 diploid cultivars analyzed in this study was preliminarily constructed (Fig. 1) and cultivars belonging to the 'Tanaka'/'Kusunoki' group, those belonging to the 'Mogi' group and the Chinese cultivar group formed three loose groups, but with no clear distinctive grouping. 'Mogi', 'Tanaka', and 'Kusunoki', which were derived from seedlings of Chinese loquat cultivars, are the current major commercial cultivars as well as the parents of many recent hybrid cultivars. These genetic relationships were reflected in the phenogram. The cultivars bred in Chiba 
Table 5. SSR genotypes of 'Kibou' and its parent cultivars.

\begin{tabular}{|c|c|c|c|c|}
\hline \multirow{2}{*}{$\begin{array}{c}\text { SSR } \\
\text { marker }\end{array}$} & \multirow[b]{2}{*}{ Origin } & \multicolumn{3}{|c|}{ Genotype $^{\mathrm{z}}$} \\
\hline & & Kibou & $\begin{array}{c}\text { 4N-Tanaka } 1 \\
\text { (female parent) }\end{array}$ & $\begin{array}{l}\text { Nagasakiwase } \\
\text { (male parent) }\end{array}$ \\
\hline $\mathrm{CH} 03 \mathrm{a} 09$ & apple & $140 / 150$ & $140 / 150$ & $134 / \underline{140}$ \\
\hline $\mathrm{CH} 03 \mathrm{~d} 07$ & apple & $306 / \underline{308}$ & $306 / 308$ & $\underline{308 / 314}$ \\
\hline $\mathrm{CH} 03 \mathrm{~d} 12$ & apple & $\underline{115 / 140}$ & $115 / 140$ & $\underline{115 / 138}$ \\
\hline $\mathrm{CH} 03 \mathrm{~g} 06$ & apple & $178 / \underline{180}$ & $178 / 178$ & $176 / \underline{180}$ \\
\hline $\mathrm{CH} 04 \mathrm{~h} 03$ & apple & $\underline{94 / 102}$ & $102 / 102$ & $\underline{94 / 94}$ \\
\hline $\mathrm{CH} 05 \mathrm{a} 04$ & apple & $185 / \underline{187}$ & $185 / 185$ & $185 / \underline{187}$ \\
\hline $\mathrm{NH} 033 \mathrm{~b}$ & pear & $\underline{184 / 196}$ & $196 / 196$ & $\underline{184 / 196}$ \\
\hline NH035a & pear & $144 / \underline{144}$ & $144 / 144$ & $\underline{144 / 144}$ \\
\hline NH204a & pear & $110 / \underline{110}$ & $110 / 110$ & $\underline{110 / 114}$ \\
\hline $\mathrm{NH} 207 \mathrm{a}$ & pear & $156 / \underline{156}$ & $156 / 156$ & $\underline{156 / 156}$ \\
\hline KA14 & pear & $175 / \underline{175}$ & $175 / 175$ & $\underline{175} / 175$ \\
\hline $\mathrm{NH} 007 \mathrm{~b}$ & pear & $129 / \underline{141 / 147}$ & $129 / 147$ & $129 / \underline{141}$ \\
\hline NH011b & pear & $\underline{174 / 184}$ & $174 / 184$ & $\underline{174 / 174}$ \\
\hline NH014a & pear & $164 / 182 / \underline{188}$ & $164 / 182$ & $135 / \underline{188}$ \\
\hline NB114a & pear & $\underline{133} / 137$ & $137 / 137$ & $\underline{133} / 133$ \\
\hline NB141b & pear & $\underline{143 / 151}$ & $143 / 151$ & $\underline{143} / 161$ \\
\hline $\mathrm{CH} 05 \mathrm{c} 06$ & apple & $107 / \underline{107}$ & $107 / 107$ & $\underline{107 / 107}$ \\
\hline $\mathrm{CH} 05 \mathrm{~g} 03$ & apple & $\underline{151 / 161}$ & $161 / 180$ & $143 / \underline{151}$ \\
\hline $\mathrm{CH} 05 \mathrm{~g} 08$ & apple & $160 / \underline{199}$ & $160 / 199$ & $164 / \underline{199}$ \\
\hline MS06c09 & apple & $141 / \underline{141}$ & $141 / 141$ & $119 / \underline{141}$ \\
\hline MS14b04 & apple & $267 / \underline{267}$ & $267 / 267$ & $\underline{267 / 267}$ \\
\hline $\mathrm{CH} 02 \mathrm{~d} 10 \mathrm{a}$ & apple & $\underline{212 / 220}$ & $220 / 220$ & $\underline{212 / 212}$ \\
\hline CH05d04 & apple & $192 / \underline{192}$ & $192 / 192$ & $\underline{192 / 192}$ \\
\hline NH015a & pear & $113 / \underline{113}$ & $113 / 113$ & $\underline{113} / 113$ \\
\hline NB105a & pear & $165 / 165$ & $165 / 165$ & $155 / \underline{165}$ \\
\hline
\end{tabular}

z Alleles derived from '4N-Tanaka 1' and 'Nagasakiwase' are indicated in italics and underlined, respectively.
Prefecture, such as 'Satomi', 'Husahikari', 'Tomihusa', and 'Husahime', formed a genetically close group in the phenogram. This may have been due to the use of common or related cultivars, such as 'Kusunoki', 'Tanaka', or their offspring, as the parents in hybridization.

More comprehensive and reliable information about the genetic diversity of the loquat will be obtained in the future by increasing the number of varieties analyzed, including indigenous varieties and Chinese cultivars.

\section{Acknowledgements}

The research was technically supported by Kazusa DNA Research Institute. The authors are grateful to Dr. Aoki (Kazusa DNA Research Institute) for valuable suggestions.

\section{Literature Cited}

Badenes, M. L., T. Canyamas, C. Romero, J. M. Soriano, J. Martinez and G. Llácer. 2003. Genetic diversityIN in European collection of loquat (Eriobotrya japonica Lindl.). Acta Hort. 620: 169-174.

Caballero, P. and M. A. Fernandez. 2002. Plenary lecture: Loquat, production and market. First international symposium on loquat (Valenia, Spain). Agenda and Abstract: 11-15.

Fukuda, S., J. Nagato, T. Yamamoto, N. Hiehata and O. Terai. 2002. Cultivar identification in loquat assessed by RAPD analysis. J. Japan. Soc. Hort. Sci. 71: 826-828 (In Japanese with English abstract).

Ichinose, I. 2002. Kajuengeigakudaihyakka 11. p. 7-10 (In Japanese). Nousanryousonbunkakyoukai, Tokyo.

Kimura, T., Y. Sawamura, K. Kotobuki, N. Matsuta, T. Hayashi, Y. Ban and T. Yamamoto. 2003. Parentage analysis in pear

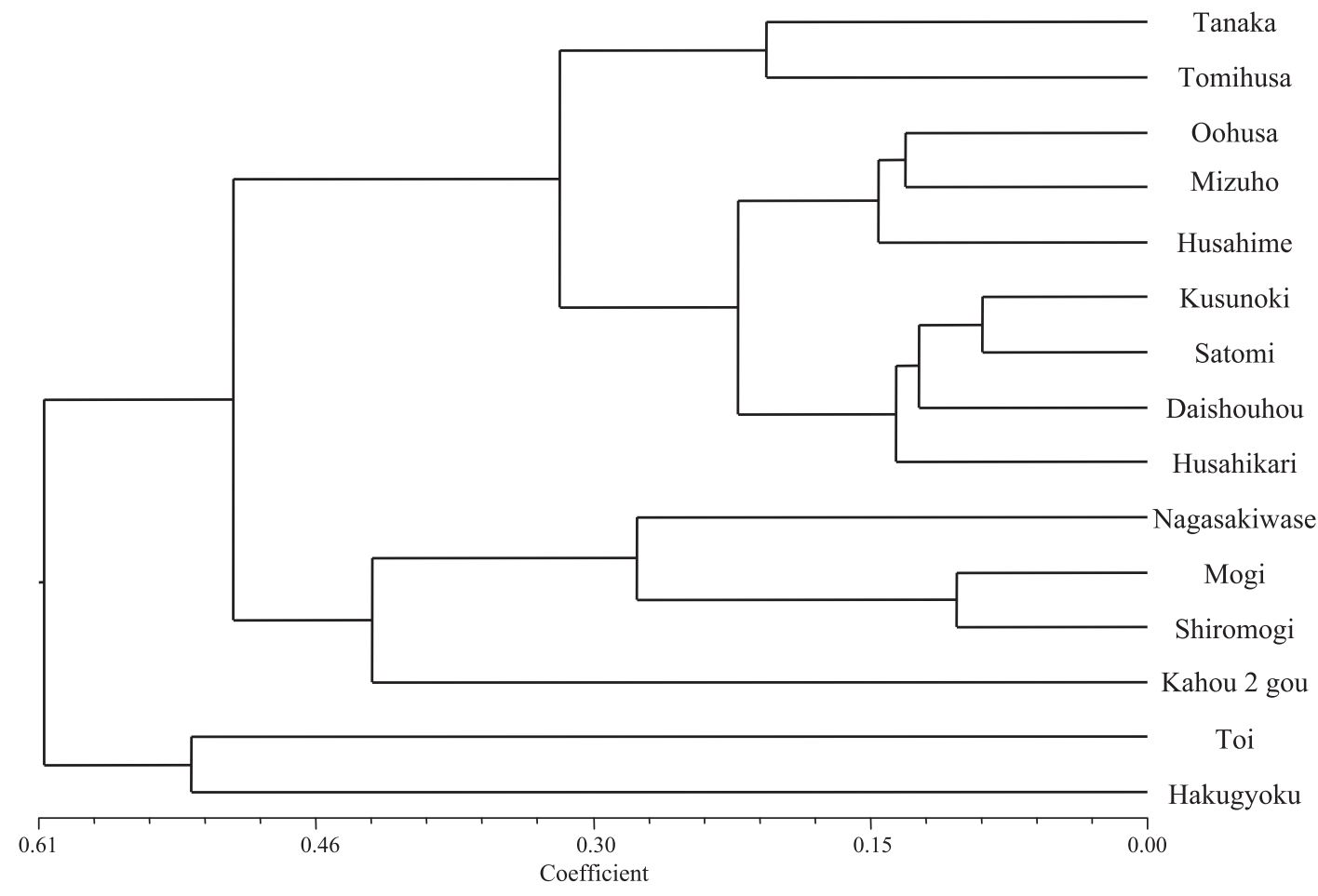

Fig. 1. Phenogram of 15 diploid loquat accessions identified by SSR genotyping generated by the UPGMA method. 
cultivars characterized by SSR markers. J. Japan. Soc. Hort. Sci. 72: 182-189.

Kimura, T., Y. Z. Shi, M. Shoda, K. Kotobuki, N. Matsuta, T. Hayashi, Y. Ban and T. Yamamoto. 2002. Identification of Asian pear varieties by SSR analysis. Breed. Sci. 52: 115-121.

Liebhard, R., L. Gianfranceschi, B. Koller, C. D. Ryder, R. Tarchini, E. Van de Weg and C. Gessler. 2002. Development and characterization of 140 new microsatellites in apple (Malus $\times$ domestica Borkh.). Mol. Breed. 10: 217-241.

Nei, M. 1972. Genetic distance between populations. Am. Nat. 106: 283-292.

Rohlf, F. J. 2005. NTSYS-pc, Numerical taxonomy and multivariate analysis system, version 2.2. Exeter Publishing, Ltd., Setauket, New York.

Sawamura, Y., T. Saito, N. Takada, T. Yamamoto, T. Kimura, T. Hayashi and K. Kotobuki. 2004. Identification of parentage of Japanese pear 'Housui'. J. Japan. Soc. Hort. Sci. 73: 511518.

Soriano, J. M., C. Romero, S. Vilanova, G. Llácer and M. L. Badenes. 2005. Genetic diversity of loquat germplasm (Eriobotrya japonica (Thunb) Lindl.) assessed by SSR markers. Genome 48: 108-114.

Vilanova, S., M. L. Badenes, J. Martínez-Calvo and G. Llácer. 2001. Analysis of loquat germplasm (Eriobotrya japonica Lindl) by RAPD molecular markers. Euphytica 121: 25-29.

Yahata, S., S. Sato, H. Ohara and H. Matsui. 2004. Induction of tetraploid in loquat with amiprofos-methyl and colchicine. Hort. Res. 3: 339-344 (In Japanese with English abstract).

Yahata, S., S. Sato, H. Ohara and H. Matsui. 2005. Differences of morphological and fruit bearing characteristics among the different ploidy plants and triploid production by crossings between diploid and triploid plants in loquat (Breeding \& Germplasm Resources). Hort. Res. 4: 379-384 (In Japanese with English abstract).

Yahata, S., S. Sato, K. Sekiyama and K. Koide. 2005. A new loquat cultivar, 'Kibou'. J. Japan. Soc. Hort. Sci. 74 (Suppl. 1): 248 (In Japanese).

Yamamoto, T., T. Kimura, Y. Sawamura, K. Kotobuki, Y. Ban, T. Hayashi and N. Matsuta. 2001. SSRs isolated from apple can identify polymorphism and genetic diversity in pear. Theor. Appl. Genet. 102: 865-870.

Yamamoto, T., T. Kimura, Y. Sawamura, T. Manabe, K. Kotobuki, T. Hayashi, Y. Ban and N. Matsuta. 2002a. Simple sequence repeats for genetic analysis in pear. Euphytica 124: 129-137.

Yamamoto, T., T. Kimura, M. Shoda, Y. Ban, T. Hayashi and N. Matsuta. 2002b. Development of microsatellite markers in the Japanese pear (Pyrus pyrifolia Nakai). Mol. Ecol. Notes 2: 14-16.

Yamamoto, T., T. Kimura, M. Shoda, T. Imai, T. Saito, Y. Sawamura, K. Kotobuki, T. Hayashi and N. Matsuta. 2002c. Genetic linkage maps constructed by using an interspecific cross between Japanese and European pears. Theor. Appl. Genet. 106: 9-18.

Yamamoto, T., T. Kimura, J. Soejima, T. Sanada, Y. Ban and T. Hayashi. 2004. Identification of quince varieties using SSR markers developed from pear and apple. Breed. Sci. 54: 239244.

Yu, D. J. 1979. Taxonomical classification of Chinese fruits. p. 309-316. In: D. J. Yu (ed.). Tropical subtropics fruit trees. 2. (In Chinese). Agricultural publisher, Chinese Beijing. 